\title{
Influence of Visual Feedback Training on the Balance and Walking in Stroke Patients
}

\author{
Kwan-Sub Lee', Han-Seong Choe'², Jae-Hong Lee ${ }^{3}$ \\ ${ }^{1}$ Kang Hospital of Daegu, Korea; ${ }^{2}$ Department of Physical Therapy, The Graduated school, Catholic University of Daegu, Korea; ${ }^{3}$ Department of \\ Physical Therapy, Daegu Health College, Korea
}

Purpose: This study aimed to evaluate changes in the balance ability of patients whose head positions were altered due to stroke. Subjects were divided into three groups to determine the effects of the training on dynamic balance and gait.

Methods: Forty-two stroke patients were enrolled. The Visual Feedback Training (VFT) group performed four sets of exercises per training session using a Sensoneck device, while the Active Range of Motion (ART) group performed eight sets per training session after receiving education from an experienced therapist. The Visual Feedback with Active Range of Motion (VAT) group performed four sets of active range of motion and two sets of visual-feedback training per session using a Sensoneck device. The training sessions were conducted three days a week for eight weeks.

Results: The comparison of changes in dynamic balance ability showed that a significant difference in the total distance of the body center was found in the VFT group $(p<0.05)$ and Significant differences were found according to the training period $(p<0.05)$. The comparison of the $10 \mathrm{~m}$ walk test showed that the main effect test, treatment period and interactions between group had statistically significant differences between the three groups $(p<0.05)$.

Conclusion: Head-adjustment training using visual feedback can improve the balance ability and gait of stroke patients. These results show that coordination training between the eyes and head with visual feedback exercises can be used as a treatment approach to affect postural control through various activities involving the central nervous system.

Keywords: Stroke, Visual Feedback, Walking, Postural Balance

\section{INTRODUCTION}

Patients with hemiplegia after stroke have balance-control problems, such as postural sway, asymmetrical weight support, damaged weight-transfer ability, and postural standing-control degradation. Since most of their daily lives are spent sitting in wheelchairs during the early stages of rehabilitation, their exercise levels are very low compared to normal healthy individuals, and their selective trunk movements and balance abilities are significantly lower in the sitting position. ${ }^{1}$ Some patients have asymmetrical posture of the head and neck due to the imbalance between muscle activity and stability and the changes in movement and stability between the head, trunk, and pelvis, as well as dominant use of the nonparetic side due

Received Nov 15, 2015 Revised Dec 22, 2015

Accepted Dec 24, 2015

Corresponding author Jae-Hong Lee

E-mail heart0630@yahoo.co.kr to the lack of balance control, thereby inducing asymmetrical bodyweight support. ${ }^{2}$ Therefore, balance on changing support-surfaces ${ }^{3}$ during the rehabilitation of stroke patients and improvement of gait function, which is the most important factor in determining functional disability in stroke patients, are two of the most important goals during the rehabilitation process for all stroke patients. ${ }^{4}$

To maintain balance effectively, it is necessary to combine the functions of the vestibular system, proprioceptors, and the visualinformation systems in the body. ${ }^{5}$ In postural control, it is important to understand the ability to gaze at visual stimuli, one of the major functions of vision, for the visual system provides information about the motion and position of the body in relation to surrounding environment, ${ }^{6}$ and vision plays an important role in de-

Copylight (C2015 The Korea Society of Physical Therapy

This is an Open Access article distribute under the terms of the Creative Commons Attribution Non-commercial License (Http:// creativecommons.org/license/by-nc/3.o.) which permits unrestricted non-commercial use, distribution, and reproduction in any medium, provided the original work is properly cited. 
termine the level of neuromuscular activity through anticipatory postural control when the body moves.

Stroke patients can lose visuospatial perception capability due to damaged function of the central nervous system, which integrates location sensory information received through visual information and proprioceptors. ${ }^{8}$ The subjective visual vertical (SVV) is determined by the internal integration of the parietal-insular-vestibular cortex. Stroke patients who have damage in this region cannot properly align their bodies straight on unstable support surfaces. ${ }^{9}$ The changed SVV causes changes in the head position of stroke patients $^{10}$ and the direction of SVV inclination is the same as that of body-trunk inclination in the standing position. ${ }^{11}$ The inclination of SVV is related to the independence of stroke patients, ${ }^{12}$ and inaccurate SVV creates balance and postural control problems. ${ }^{13}$

A trajectory-tracking task, one of the visual-feedback training methods widely used in balance training after stroke, is used for patients who have damage to quantitative movement control, such as motor coordination during single-joint movements. ${ }^{14}$ It is also used to investigate the effect of the damage to the central nervous system through accurate trajectory-tracking. ${ }^{15}$ However, existing studies have focused only on balance or postural control training using the lower and upper extremities through virtual reality programs or Wii games on monitors.

This study conducted an experiment in which patients followed specific trajectories with their eyes using the Sensoneck device in the sitting position, tracking the trajectory with a laser-pointer flashlight attached to the head and aimed at a board in order to determine whether coordination ability was increased through head movements and eye-tracking and whether there was a correlation between the head/neck area and other body parts. It also aimed to determine the effect of changing postural alignment and the mobility of neuromuscular activities via neck exercises on the postural control of stroke patients by emphasizing motor-control therapy for coordination between the head and neck.

\section{METHODS}

\section{Study subjects}

This study selected 42 patients, who understood the objective and participated voluntarily, from among stroke patients hospitalized at K Hospital in Daegu from March to April 2014. All subjects were diagnosed with stroke via computed tomography (CT) or magnetic resonance imaging $(\mathrm{MRI})$, and their ages ranged from 16 to 65 years. Within three months after stroke onset, neurologic recovery progresses quickly so patients who were still in that period were excluded, while only patients with no severe cognitive or consciousness disorders and who could follow three-step commands were selected. Patients with visual problems, such as ophthalmodonesis or diplopia, or brainstem and cerebellar symptoms (vestibular dysfunction), were also excluded from the study, as were patients with receptive aphasia and hemi-neglect. All study subjects were fully informed about the study objectives and methods prior to the experiment, and voluntary consent was received from each participant. No significant differences in homogeneity and normality tests were found between the groups ( $p>0.05)$ (Table 1).

\section{Measurement tools and procedures}

\section{1) Measuring tools}

The Sensoneck device of the TerapiMaster was used to train eyehead coordination. This device aims to test and exercise the neck

Table 1. General characteristics of the subjects

\begin{tabular}{|c|c|c|c|c|}
\hline Variables & VFT Group $(n=14)$ & ART Group $(n=14)$ & VAT Group $(n=14)$ & $\mathrm{p}$ \\
\hline Gender (male/female, n) & $11 / 3$ & $11 / 3$ & $12 / 2$ & \\
\hline Age (yr) & $55.57 \pm 2.03$ & $58.57 \pm 4.40$ & $55.43 \pm 3.39$ & 0.77 \\
\hline Height (cm) & $166.86 \pm 1.50$ & $167.42 \pm 2.33$ & $169.14 \pm 2.21$ & 0.71 \\
\hline Weight (kg) & $64.64 \pm 1.95$ & $67.92 \pm 4.41$ & $64.71 \pm 2.96$ & 0.72 \\
\hline Time since stroke(month) & $8.07 \pm 0.84$ & $9.50 \pm 0.79$ & $8.00 \pm 1.10$ & 0.44 \\
\hline Paretic side (right/left, $n$ ) & $5 / 9$ & $8 / 6$ & $7 / 7$ & \\
\hline Type of stroke (infarction/hemorrhage, n) & $7 / 7$ & $9 / 5$ & $8 / 6$ & \\
\hline
\end{tabular}

Mean \pm SD.

VFT: Visual feedback training, ART: Active ROM training, VAT: Visual feedback training + Active ROM training. ${ }^{*} p<0.05$. 
joints and deep muscles. It consists of a headset mounted with a laser pointer, and a drawing board. When the patient wears the headset, the laser is automatically displayed on the drawing board. The patient moves the beam along the lines of the drawing board, increasing muscle strength in the neck and stabilizing the neck muscles. By controlling point-movement speed, exercise difficulty can be controlled.

To measure static and dynamic abilities, the Good Balance System Ver 3.06 (METITER, USA) was employed. This machine has an inclinable foothold that can measure vertical forces applied to the heel. In our study, the static balance ability was measured by the center of pressure (COP) while the patient looked forward while in the standing position with a 4 -inch gap between the feet. The dynamic balance ability was measured by a method in which the patients moved their COP in the correct order along a path displayed on the computer screen. A 10 -m walk test with proven reliability and validity in many other studies was modified to measure the gait ability of the patients by having them perform three tasks randomly. A mean value was calculated after obtaining three measurements. ${ }^{16}$ The three tasks were as follows:

A. Walk upright with a straight head.

B. Walk while rotating the head from one side to the other.

C. Walk while lifting the head from the top to the bottom.

Each task was performed twice while walking, with a one-minute rest between tasks.

\section{2) Measurement method}

Prior to the experiment, the objectives and test methods of this study were explained to the 42 stroke patients who voluntarily consented to the experiment, and a therapist did demonstrations of all tasks. To determine the effect of visual-feedback interventions on stroke patients according to the training period and the number of sessions, the subjects were divided into three groups. They were randomly assigned into a visual-feedback training (VFT) group, an active range-of-motion training (ART) group, and a group that combined visual feedback with active range-of-motion training (VAT). All patients performed comprehensive rehabilitation therapy, including physiotherapy, occupational therapy, language therapy, and cognitive therapy. If needed, walking aids were used for balance training.

For the VFT group, training was carried out by means of the sen- soneck device. The subjects were positioned $2.5 \mathrm{~m}$ away from the picture board, and headsets with laser points were put on them. Trajectories on the picture board were divided into a vertical line, a horizontal line, an oblique line, a figure of eight, and an oval. And the posture of sitting up straight was used as the training posture. The first set was composed of the horizontal line, the vertical line, the oblique line, the figure of eight, and the oval. Four sets were performed a day, with a three-minute rest between sets. The training was carried out for eight weeks, four sets a day and three days a week. For the ART group, a trained therapist gave a demonstration first, and then the patients were given education. As for the active range of motion of neck, flexion and extension, turning to the left and turning to the right, extension to the left, flexion to the right, extension to the right, flexion to the left, and head turn motion were performed in random sequence. Eight sets were performed a day, with a one-minute break between sets. The training was carried out for eight weeks, eight sets a day and three days a week. For the VAT group, the active range of motion of neck was carried out in random sequence for eight weeks, four sets a day and three days a week; and the visual feedback training was carried out in random sequence, tracing trajectories on the picture board. And the training was carried out for eight weeks, two sets a day and three days a week.

Goodbalance was used to measure balance ability. In the standing position, sway speeds in the COP A-P and M-L directions were measured with the eyes open and closed, respectively. In addition, the travel distance of the center of weight and the total travel distance were measured. To ensure the accuracy of the measurement of balance and walking, a single examiner obtained three measurements and a mean value was used. Three measurements for each subject were obtained prior to the experiment, after four weeks, and after eight weeks, and mean values were calculated.

\section{Statistics}

The data acquired in this study were statistically processed using SPSS version 18.0. The general characteristics of the subjects were analyzed using one-way ANOVA, and two-way repeated ANOVA was used to compare differences between balance and walking abilities prior to the experiment, after four weeks, and after eight weeks for each group. For post-hoc comparison, least significant difference was calculated. The statistical significance level of $\alpha$ was set at $\mathrm{p}<0.05$. 


\section{RESULTS}

\section{Comparison of dynamic balance ability \\ 1) Comparison of changes in total distance}

Main effect tests showed no statistically significant differences between the three groups $(\mathrm{p}>0.05)$ but between the training periods (prior to experiment, after four weeks, and after eight weeks) $(\mathrm{p}<0.05)$. Furthermore, interactions between the groups and between the time before and after the experiment (prior to the experiment, after four weeks, and after eight weeks) showed no statistically significant differences $(\mathrm{p}>0.05)$ (Table 2).

\section{2) Comparison of changes in M-L distance}

Main effect tests showed statistically significant differences between the three groups $(\mathrm{p}<0.05)$ and between the training periods (prior to experiment, after four weeks, and after eight weeks) $(\mathrm{p}<0.05)$. Furthermore, interactions between groups and between the time before and after the experiment (prior to the experiment, after four weeks, and after eight weeks) also showed statistically significant differences $(\mathrm{p}<0.05)$ (Table 3).

\section{3) Comparison of changes in A-P distance}

Main effect tests showed no statistically significant differences between the three groups $(p>0.05)$ but between the training periods (prior to experiment, after four weeks, and after eight weeks) $(\mathrm{p}<0.05)$. Furthermore, interactions between groups and between the time before and after the experiment (prior to the experiment, after four weeks, and after eight weeks) showed no statistically significant differences $(\mathrm{p}>0.05)$ (Table 4$)$.

\section{Comparison of changes in $10 \mathrm{~m}$ walking speed between groups}

Main effect tests showed statistically significant differences between the three groups $(\mathrm{p}<0.05)$ and between the training periods (prior to experiment, after four weeks, and after eight weeks) $(\mathrm{p}<0.05)$. Furthermore, interactions between the groups and between the time before and after the experiment (prior to the experiment, after four weeks, and after eight weeks) also showed statistically signifi-

Table 2. A comparison of total distance during the intervention on each group

(Unit: $\mathrm{mm}$ )

\begin{tabular}{|c|c|c|c|c|c|c|}
\hline & \multirow{2}{*}{ Pre-test $(\mathrm{M} \pm \mathrm{SD})$} & \multirow{2}{*}{4 week $(M \pm S D)$} & \multirow{2}{*}{8 week $(M \pm S D)$} & \multicolumn{3}{|c|}{$F(p)$} \\
\hline & & & & Group & Period & Group*Period \\
\hline VFT Group & $1,602.88 \pm 407.02$ & $1,522.38 \pm 528.42$ & $1,434.12 \pm 352.80$ & & & \\
\hline ART Group & $1,771.15 \pm 508.13$ & $1,761.40 \pm 549.99$ & $1,671.50 \pm 524.99$ & $1.34(0.27)$ & $5.45(0.01 *)$ & $1.16(0.33)$ \\
\hline VAT Group & $1,757.27 \pm 676.68$ & $1,754.25 \pm 652.91$ & $1,667.32 \pm 584.29$ & & & \\
\hline
\end{tabular}

VFT: Visual feedback training, ART: Active ROM training, VAT: Visual feedback training + Active ROM training. ${ }^{*} p<0.05$.

Table 3. A comparison of M-L distance during the intervention on each group

(Unit: mm)

\begin{tabular}{|c|c|c|c|c|c|c|}
\hline & \multirow{2}{*}{ Pre-test $(M \pm S D)$} & \multirow{2}{*}{4 week $(M \pm S D)$} & \multirow{2}{*}{8 week $(M \pm S D)$} & \multicolumn{3}{|c|}{$F(p)$} \\
\hline & & & & Group & Period & Group*Period \\
\hline VFT Group & $990.58 \pm 341.77$ & $910.47 \pm 287.05$ & $758.86 \pm 176.01$ & & & \\
\hline ART Group & $1,220.33 \pm 390.75$ & $1,154.50 \pm 474.98$ & $1,131.44 \pm 347.56$ & $3.89\left(0.03^{*}\right)$ & $4.17\left(0.04^{*}\right)$ & $0.93(0.42)$ \\
\hline VAT Group & $1,316.21 \pm 581.48$ & $1,199.85 \pm 633.61$ & $1,143.39 \pm 487.82$ & & & \\
\hline
\end{tabular}

VFT: Visual feedback training, ART: Active ROM training, VAT: Visual feedback training + Active ROM training.

${ }^{*} p<0.05$.

Table 4. A comparison of A-P distance during the intervention on each group

(Unit: $\mathrm{mm}$ )

\begin{tabular}{|c|c|c|c|c|c|c|}
\hline & \multirow{2}{*}{ Pre-test $(\mathrm{M} \pm \mathrm{SD})$} & \multirow{2}{*}{4 week $(\mathrm{M} \pm \mathrm{SD})$} & \multirow{2}{*}{8 week $(\mathrm{M} \pm \mathrm{SD})$} & \multicolumn{3}{|c|}{$F(p)$} \\
\hline & & & & Group & Period & Group*Period \\
\hline VFT Group & $1,161.63 \pm 473.37$ & $1,016.31 \pm 398.82$ & $928.72 \pm 232.79$ & & & \\
\hline ART Group & $1,268.47 \pm 398.28$ & $1,208.28 \pm 399.53$ & $1,178.76 \pm 326.36$ & $1.87(0.17)$ & $4.84\left(0.02^{*}\right)$ & $1.84(0.14)$ \\
\hline VAT Group & $1,352.72 \pm 523.98$ & $1,255.50 \pm 396.67$ & $1,248.95 \pm 501.07$ & & & \\
\hline
\end{tabular}

VFT: Visual feedback training, ART: Active ROM training, VAT: Visual feedback training + Active ROM training.

${ }^{*} p<0.05$. 


\begin{tabular}{|c|c|c|c|c|c|c|}
\hline & \multirow{2}{*}{ Pre-test $(\mathrm{M} \pm \mathrm{SD})$} & \multirow{2}{*}{4 week $(M \pm S D)$} & \multirow{2}{*}{8 week $(\mathrm{M} \pm \mathrm{SD})$} & \multicolumn{3}{|c|}{$F(p)$} \\
\hline & & & & Group & Period & Group*Period \\
\hline VFT Group & $22.57 \pm 2.24$ & $20.64 \pm 2.76$ & $18.64 \pm 2.37$ & $3.31\left(0.04^{*}\right)$ & $209.07\left(0.00^{*}\right)$ & $40.63\left(0.00^{*}\right)$ \\
\hline ART Group & $23.28 \pm 2.97$ & $23.00 \pm 2.96$ & $22.57 \pm 2.93$ & & & \\
\hline VAT Group & $22.93 \pm 2.02$ & $22.07 \pm 1.73$ & $21.14 \pm 1.61$ & & & \\
\hline
\end{tabular}

VFT: Visual feedback training, ART: Active ROM training, VAT: Visual feedback training + Active ROM training. ${ }^{*} p<0.05$.

cant differences $(\mathrm{p}<0.05)$ (Table 5).

\section{DISCUSSION}

Many stroke patients experience changes in head position, but most rehabilitation focuses on functional treatment and balance training for the trunk, arms, and legs, ignoring the correlation between head position, postural evaluation, and balance control. Thus, it is necessary for hemiplegic stroke patients to undergo evaluation and treatment of the head and neck. In particular, studies on the relationship between incorrect neck posture and balance control in stroke patients should be conducted.

This study aimed to identify the effect of improved neck posture through head/neck exercises in stroke patients and to identify balance-control problems that can occur due to bad posture, thereby providing foundational data that are useful for balance-control exercise programs for these patients.

Anabela et al. ${ }^{17}$ reported that proprioceptive afferent inputs from neck muscles play an important role in postural control, and Anders, Brose et al. ${ }^{18}$ reported that three-dimensional head control exercise is effective in neuromuscular control ability, the facilitation of proprioception, increase in balancing ability, and trunk stabilization. In addition, Sackley and $\operatorname{Lincoln}^{19}$ reported that visual feedback training is effective in gait symmetry in stroke patients, and Bonan et al. ${ }^{20}$ reported that visuo-perceptual training is effective in the improvement of balance and gait in stroke patients, showing agreement with antecedent research.

Voluntary head movements in the standing position or during walking are frequently performed in daily living and are accompanied by eye movement to examine the surrounding environments or to visually trace external stimulation. ${ }^{21}$ The three tasks during walking were conducted in this study using a modified $10 \mathrm{~m}$ walking test. On this test, the VFT group showed a significant difference according to training period and in comparison to the other groups.
Walker et al. ${ }^{22}$ and Dault et al. ${ }^{23}$ applied visual feedback to patients who had damage to the central nervous system, and identified body-movement travel and spatial disorientation in the standing position, thereby improving postural control ability. Laufer et al. ${ }^{24}$ reported that the visual-feedback effect played an important role in the postural control of patients with balance control disorders. The present study also showed results similar to previous studies, demonstrating a significant difference with the visual feedback training. Meesen et al. ${ }^{25}$ claimed that rotation and diagonal head movements affect coordination between the lower and upper extremities so that various periodic coordination activities that depend on head stability can influence postural balance, and reported that eye-gazing exercises can induce concentration strength and affect postural balance. Jamet et al. ${ }^{26}$ reported that independent walking can be facilitated based on postural control through stability in the standing position and with unbiased weight distribution.

Thus, various activities through coordinated movements between the eyes and head can be one of the therapeutic approaches to affecting the postural control of stroke patients. If visual-feedback training such as trajectory tracking is applied to improve motor functions and balance-maintaining ability for stroke patients, their functional daily living performance will be improved.

The limitation of this study was that no measurement of saccade movements was conducted due to the focus on eye movements and the lack of studies on the effect of increased head coordination on coordination between the upper and lower extremities. In future studies, it will be necessary to investigate the effect of distal-portion activities, such as head movements, on the stability of the proximal portions of the body and the coordination of the extremities.

\section{REFERENCES}

1. Kim GH, Choe HS, Lee HI et al. The effects of scapular stabilization exercising on dynamic standing balance in stroke patients. J Korean Soc Phys Ther. 2014;2(1):15-20. 
2. Lee KS, Choe HS, Lee BJ. Variations in stroke patients' muscle activity during head rotation in non-paretic-side weight bearing. J Kor Phys Ther. 2015:27(3):159-63.

3. Shin YI, Yang SH Kim JY. Clinical feasibility of wearable robot orthosis on gait and balance ability for stroke rehabilitation: A case study. J Kor Phys Ther. 2015:27(2):124-7.

4. Kim JH. Relationship between gait symmetry and functional balance, walking performance in subjects with stroke. J Korean Soc Phys The. 2014;2(1):1-8.

5. Lindy JF, Janssen, Lonneke L et al. Directional effects of biofeedback on trunk sway during gait tasks in healthy young people. Gait \& Posture. 2009;29:557-81.

6. Shumway-Cook A, Woollacott MH. Motor control: Translating research into clinical practice. Lippincott Williams \& Wilkins, 2007.

7. Wade MG, Jones $\mathrm{G}$. The role of vision and spatial orientation in the maintenance of posture. Phys Ther. 1997;77(6):619-28.

8. Yelnik AP, Lebreton FO, Bonan IV et al. Perception of verticality after recent cerebral hemispheric stroke. Stroke. 2002;33(9):2247-53.

9. Perennou DA, Leblond C, Amblard B et al. The polymodal sensory cortex is crucial for controlling lateral postural stability: Evidence from stroke patients. Brain Res Bull. 2000;53(3):359-65.

10. Guerraz M, Sakellari V, Burchill P et al. Influence of motion parallax in the control of spontaneous body sway. Exp Brain Res. 2000;131(2):24452.

11. Snowdon N, Scott O. Perception of vertical and postural control following stroke: A clinical study. Physiotherapy. 2005;91(3):165-70.

12. Kerkhoff G. Multimodal spatial orientation deficits in left-sided visual neglect. Neuropsychologia. 1999;37(12):1387-405.

13. Brandt T, Dieterich M. Perceived vertical and lateropulsion: Clinical syndromes, localization, and prognosis. Neurorehabil Neural Repair. 2000;14(1):1-12.

14. Petrofsky JS, Petrofsky D. A simple device to assess and train motor coordination. J Med Eng Technol. 2004;28(2):67-73.

15. Carey JR, Anderson KM, Kimberley TJ et al. Fmri analysis of ankle movement tracking training in subject with stroke. Exp Brain Res. 2004; 154(3):281-90.

16. Uthaikhup S, Sunkarat S, Khamsaen K et al. The effects of head movement and walking speed on gait parameters in patients with chronic neck pain Manual Therapy. 2014;19:137-41.

17. Anabella G, Silva, Mark I, Johnson. Does forward head posture affect postural control in human healthy volunteers? Gait \&Posture. 2013:38: 352-53.

18. Anders C, Brose G. Activation characteristics of trunk muscles during whole body tilt with unsupported trunk ,European Journal of Applied Physiology. 2005;11:195-200.

19. Bonan I, Leman M, Legargasson J et al. Evolution of subjective visual vertical perturbation after stroke. Neurorehabilitation and neural repair. 2006;20(4):484-91.

20. Sackley CM, Lincoln NB. Single blind randomized controlled trial of visual feedback after stroke: Effects on stance symmetry and function. Disabil Rehabil. 1997;19(12):536-46.

21. Anouk L, Nicole P, Joyce F. Postural adjustments to voluntary head motions during standing are modified following stroke. Clinical Biomechanics. 2003;18:832-42.

22. Walker C, Brouwer BJ, Culham EG. Use of visual feedback in retraining balance following acute stroke. Phys Ther. 2000;80(9):886-95.

23. Dault MC, de Haart M, Geurts AC et al. Effects of visual center of pressure feedback on postural control in young and elderly healthy adults and in stroke patients. Human movement science. 2003;22(3):221-36.

24. Laufer Y, Sivan D, Schwarzmann R et al. Standing balance and functional recovery of patients with right and left hemiparesis in the early stages of rehabilitation. Neurorehabil Neural Repair. 2003;17(4):207-13.

25. Meesen R, Levin O, Wenderoth $\mathrm{N}$ et al. Head movements destabilize cyclical in-phase but not anti-phase homologous limb coordination in humans. Neuroscience letters. 2003;340(3):229-33.

26. Jamet M, Deviterne D, Gauchard GC et al. Age-related part taken by attentional cognitive processes in standing postural control in a dual-task context. Gait \& posture. 2007;25(2):179-84. 